\title{
Flow Stress Modelling and Microstructure Development during Deformation of Metallic Materials
}

\author{
Johannes Kreyca ${ }^{1, a}$, Ernst Kozeschnik ${ }^{1, b^{*}}$ \\ ${ }^{1}$ Institute of Materials Science and Technology, TU Wien, \\ Getreidemarkt 9, Vienna, 1060, Austria \\ ajohannes.kreyca@tuwien.ac.at, bernst.kozeschnik@tuwien.ac.at
}

Keywords: Large deformation, stage IV, state-parameter

\begin{abstract}
A constant strain hardening rate is characteristic for large strain deformation at low temperatures and often observed during wire drawing. This stage of deformation, in the following referred to as stage IV, is determined by the microstructural evolution of dislocation cells. At elevated temperatures, rapid stress saturation is typically reached and no stage IV behavior is observed. This behavior is modelled in the present work, following the concept of state-parameter based plasticity, evolving dislocation density and subgrain formation as functions of strain rate, strain and temperature. It is demonstrated that the temperature dependence of state parameters at different deformation stages is closely related. The present model is compared to a series of compression tests carried out on a Gleeble 1500 thermo-mechanical simulator. EBSD micrographs of the same material reveal the microstructural evolution during plastic deformation. It is shown experimentally that the transition from cell forming behavior to subgrain formation correlates well with the disappearance of stage IV and the overall change in the dominant mechanism for overcoming obstacles. In combination with thermally activated yield stress prediction, this model, recently implemented in the software package MatCalc, offers a powerful tool for flow-curve simulation.
\end{abstract}

\section{Introduction}

Large strain deformation associated with Stage IV hardening is characteristic for wire drawing. Stage IV behavior is characterized by a constant strain hardening rate up to very high strains. Though it was experimentally first characterized by Langford and Cohen [1] for iron in tension experiments for previously wire drawn specimen, it is normally not observed in tension experiments since strains are too low for its occurrence. The experimental method of choice is, therefore, torsion or compression testing. Stage IV hardening is typically observed at low and intermediate temperatures where the dominant deformation mechanism is cross slip. The strain-hardening rate is temperature and strain-rate dependent but only weakly dependent on alloy composition, as shown by Ryen et al. [2] for AlMg-alloys and by Kocks [3] for Silver and Ni-Mo alloys.

In most alloy systems, deformation is governed by the motion of dislocations. Hardening occurs due to obstacles that hinder this motion. The frequency or density of these obstacles, together with their individual ability to pin dislocations, characterizes the magnitude of the individual strength contribution. For strain hardening, blocked dislocations serve as obstacles. Thus, the actual microstructure formed during previous deformation characterizes the stress response of the material. These blocked dislocations might be evenly distributed (forest dislocations) or concentrated into cells or subgrains.

\section{Physical Models}

Physical models aim to model the dislocation evolution based on state parameters, such as, an average dislocation density or a subgrain diameter. In this context, the Kocks-Mecking model (KMmodel) is a well-established [4,5] one-parameter model for the average dislocation density and takes the form 


$$
\frac{\mathrm{d} \rho}{\mathrm{d} \varepsilon}=\mathrm{h}_{1} \sqrt{\rho}-\mathrm{h}_{2} \rho=\frac{\mathrm{M}}{\mathrm{bA}} \sqrt{\rho}-2 \mathrm{BM} \frac{\mathrm{d}_{\text {crit }}}{\mathrm{b}} \rho
$$

With

$$
\mathrm{d}_{\mathrm{crit}}=\frac{\mathrm{Gb}^{4}}{2 \pi(1-v) \mathrm{Q}_{\mathrm{vac}}}
$$

as the annihilation distance between two dislocations [6] and

$$
\sigma=\alpha \mathrm{bGM} \sqrt{\rho}
$$

as the Taylor equation, where $Q_{\text {vac }}$ is the vacancy formation energy, $b$ is the Burgers vector, $M$ is the Taylor factor, $G$ is the shear modulus, and $\alpha$ is a numerical constant close to unity. $A$ and $B$ are numerical constants. Eq. (1) leads to saturation upon integration with zero strain hardening rate after a finite amount of strain. Therefore, additional state-parameters are introduced $[7,8]$ for taking stage IV into account.

Different rules of mixture for the impact of the individual state-parameter on the macroscopic stress were introduced into Eq. (2), for a review see [9]. In the following, a straightforward approach is adopted, based on another feature of stage IV: It is widely accepted [10] that the stage IV hardening rate $\theta_{I V}=d \sigma_{I V} / d \varepsilon$ is related to the stress at the beginning of stage IV through the simple linear relation

$$
\theta_{\mathrm{IV}}=\mathrm{c}_{\mathrm{IV}} \sigma_{\mathrm{IV}}
$$

Combining eqs. (1) and (2) delivers

$$
\theta=\mathrm{k} \sigma-\mathrm{d}
$$

for stage III, where $k=-h_{2} / 2$ and $d=h_{1} g_{1} / 2$ are constants related to $h_{1}$ and $h_{2}$ in Eq. (1). At one intersection, Eqs. (3) and (4) are fulfilled leading to

$$
\theta_{\mathrm{IV}}=\frac{1}{\mathrm{~d}}\left(\frac{\mathrm{k}}{\mathrm{c}_{\mathrm{IV}}}-1\right)
$$

Thus, the temperature and strain-rate dependence of stage IV is characterized by the thermally activated behavior of stage III and its absolute value is defined by a constant $c_{\mathrm{IV}}$.

\section{Experimental}

Experiments are carried out on a Gleeble 1500 thermo-mechanical simulator. Cylindrical specimens, made from an A6061 aluminium alloy, are tested in the dimensions of $15 \times 10 \mathrm{~mm}$. The chemical composition is summarized in Table 1. 

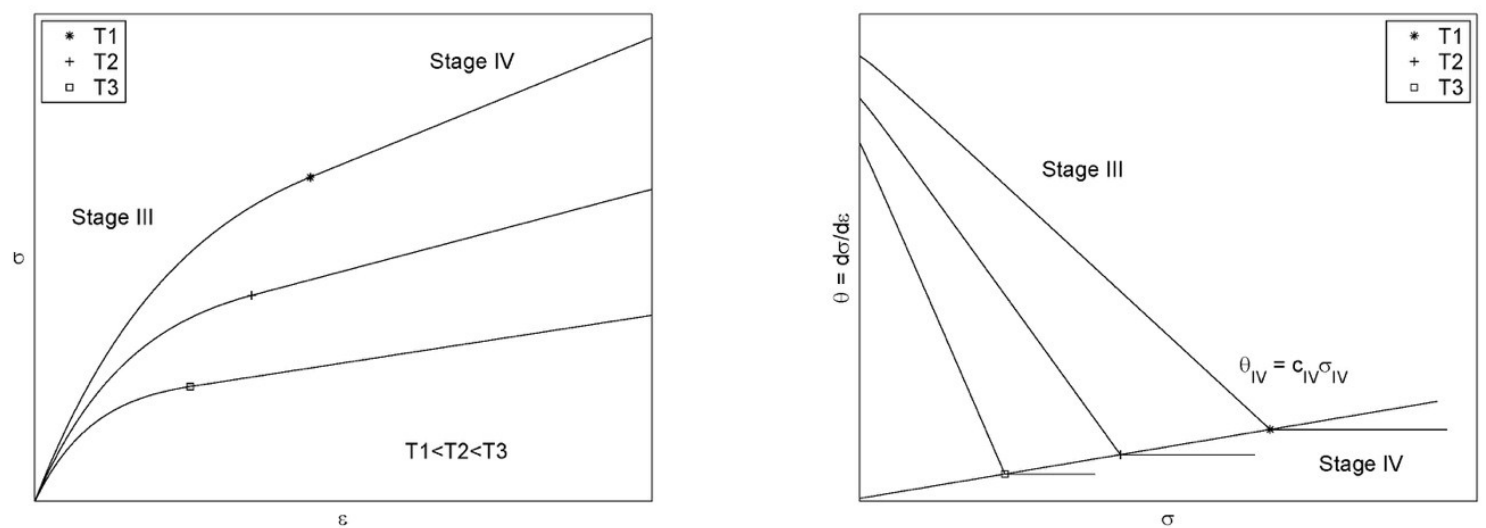

Fig. 1. (a) Schematic view of stress-strain curves for three different temperatures (b) Strain hardening rate over stress (Kocks-Plot) for data in (a)

Table 1. Chemical Composition

\begin{tabular}{lcccccccccc}
\hline Element & $\mathrm{Al}$ & $\mathrm{Si}$ & $\mathrm{Fe}$ & $\mathrm{Cu}$ & $\mathrm{Mn}$ & $\mathrm{Mg}$ & $\mathrm{Cr}$ & $\mathrm{Ni}$ & $\mathrm{Zn}$ & $\mathrm{Ti}$ \\
\hline Concentration [\%] & 97.35 & 0.69 & 0.45 & 0.23 & 0.11 & 0.85 & 0.18 & $<0.1$ & $<0.1$ & $<0.1$ \\
\hline
\end{tabular}

The specimens are solution heat treated for $4 \mathrm{~h}$ at $540{ }^{\circ} \mathrm{C}$, water quenched and artificially aged at $170{ }^{\circ} \mathrm{C}$. The samples were tested at a strain rate of $0.01 \mathrm{~s}^{-1}$ and five different temperatures. The experimental values are the average of three experiments. EBSD micrographs were taken from samples at $25{ }^{\circ} \mathrm{C}, 150{ }^{\circ} \mathrm{C}, 200{ }^{\circ} \mathrm{C}$ and $350{ }^{\circ} \mathrm{C}$ using the steps for grinding and polishing [11] as summarized in Table 2.

Table 2. Grinding and polishing

\begin{tabular}{lccc}
\hline Step & Polishing Disc & Grit $[\mu \mathrm{m}]$ & Time [min] \\
\hline Grinding & Abrasive Paper & 500 & 2 \\
Polishing & Struers MD Largo & 9 & 15 \\
Polishing & Struers MD Dur & 6 & 25 \\
Polishing & Struers MD Mol & 3 & 30 \\
OPS & Struers MD-Chem & 1 & 15 \\
Cleaning & Struers MD-Chem & 1 & 3 \\
\hline
\end{tabular}

The EBSD investigations are carried out using an FEI Quanta 200 FEG. The data analysis was conducted with EDAX Orientation Imaging Microscopy (OIM) Data Analysis. Table 3 shows the parameters used in the simulation.

Table 3. Parameters used in modelling

\begin{tabular}{llll}
\hline Abbreviation & Name & Value & Unit \\
\hline$b$ & Burgers vector & $2.8510^{-10}$ & {$[\mathrm{~m}]$} \\
$M$ & Taylor factor & 3.06 & {$[-]$} \\
$G$ & Shear modulus & $29438.4-15.052 \cdot \mathrm{T}$ & {$[\mathrm{MPa}]$} \\
$\dot{\varepsilon}$ & Strain rate & 0.01 & {$[-]$} \\
$v$ & Poisson's ratio & 0.3 & {$[-]$} \\
$Q^{f v}$ & Vacancy formation energy & 0.67 & {$[\mathrm{eV}]$} \\
$\alpha$ & Coefficient for inner dislocation density & 0.5 & {$[-]$} \\
& strengthening & & \\
$A$ & Dislocation production parameter & $26,26,27.6,33.38,35.07$ & {$[-]$} \\
$B$ & Dislocation annihilation parameter & $3.98,4.3,4.76,4.95,6.74$ & {$[-]$} \\
$c_{\mathrm{IV}}$ & Stage IV coupling coefficient & 1.5 & {$[-]$} \\
\hline
\end{tabular}




\section{Results}

Fig. 3(a) shows the measured values together with simulation results. The values for $A$ and $B$ were fitted (see Table 3 ). It should be mentioned that $A$ and $B$ are temperature and strain rate dependent, according to thermal activation theory [12], and can conveniently be modelled within this framework. A closer discussion of this aspect is out of the scope of this work, however. The present work is focused on the relation between stage III and IV behavior showing that no additional fitting for stage IV is needed for the model. Fig. 3(b) shows the resulting evolution of the dislocation density for the data shown in Fig. 2(a).

Fig. 2 presents the EBSD micrographs for $25{ }^{\circ} \mathrm{C}, 150{ }^{\circ} \mathrm{C}, 200{ }^{\circ} \mathrm{C}$ and $350{ }^{\circ} \mathrm{C}$ deformation temperature at a strain of 0.2 . At low temperatures, corresponding to the occurrence of stage IV, a geometrically ordered cell-structure is observed. It can be shown that these structures are oriented relative to the rolling direction $[13,11]$ and that the spacing between alternating orientations is a function of temperature, strain rate and strain. At $350{ }^{\circ} \mathrm{C}$, a totally different microstructure occurs. Due to vacancy assisted climb processes, dislocations can now move out of their slip plane and organize themselves in the form of energetically favorable subgrains. These subgrains, with their tendency to grow in size at higher temperature, lead to the saturation of the stage IV hardening rate and, thus, to zero strain hardening for intermediate temperatures.

(a)

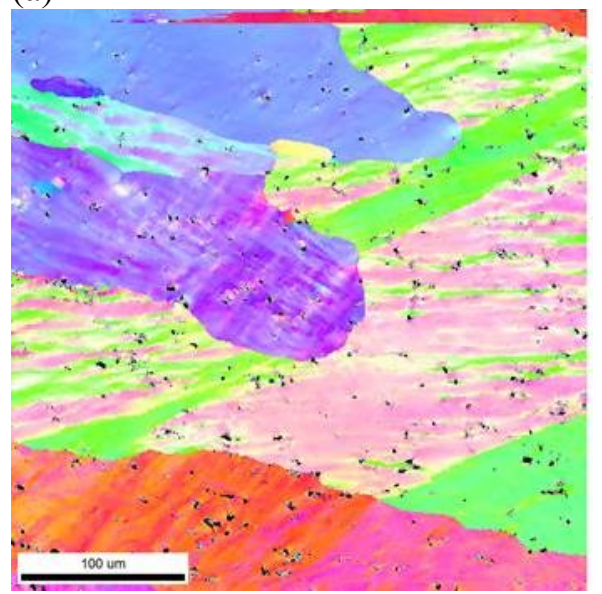

(c)

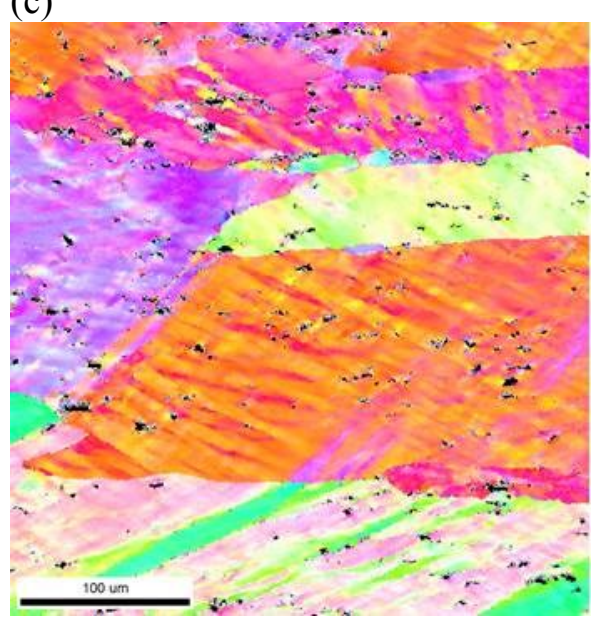

(b)

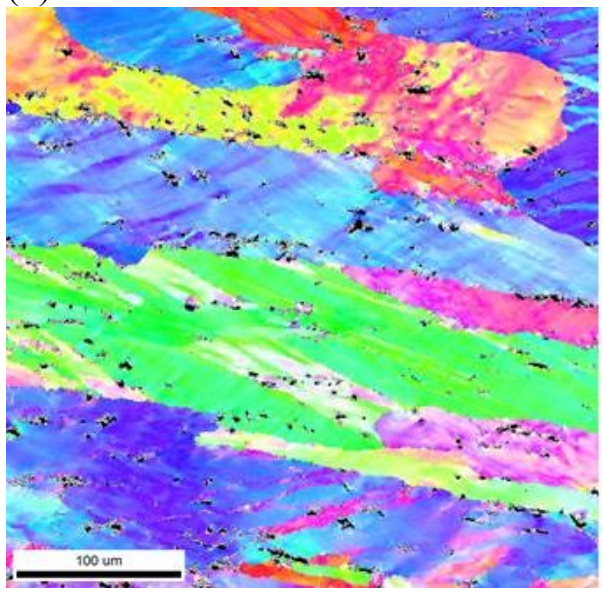

(d)

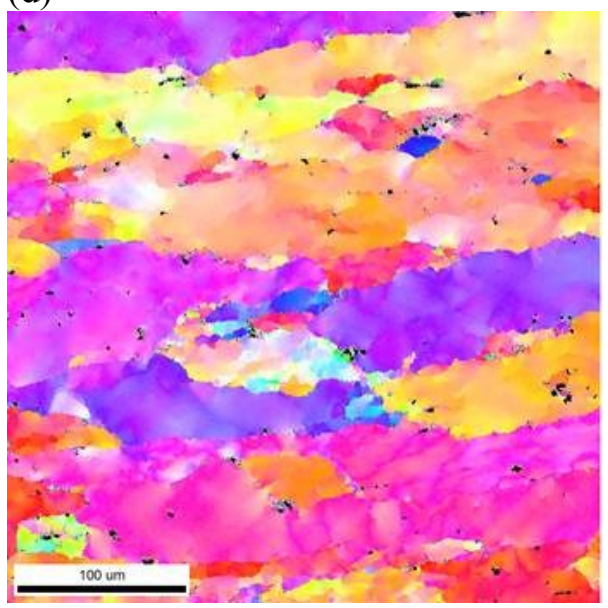

Fig. 2. EBSD micrographs for (a) $25^{\circ} \mathrm{C}$, (b) $150^{\circ} \mathrm{C}$, (c) $200{ }^{\circ} \mathrm{C}$ and (d) $350^{\circ} \mathrm{C}$ at a strain of 0.2 

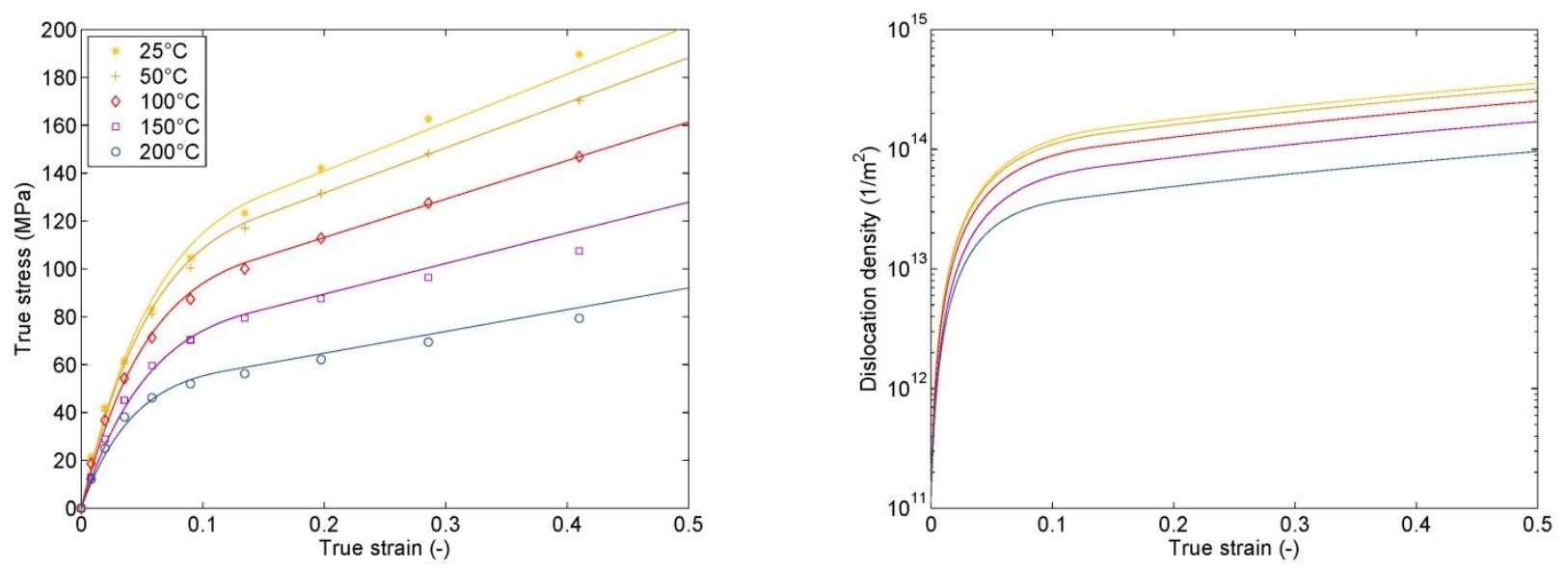

Fig. 3. (a) Experimental (symbols) and simulated (lines) stress-strain curves for five different temperatures (b) corresponding simulated dislocation density evolution

\section{Conclusion}

A physically-based one-parameter model based on the Kocks-Mecking approach for the description of stage III and IV deformation behavior is presented. It is shown that temperature and strain-rate dependent behavior of stage IV is readily deduced from stage III thermal activation, indicating that the physical mechanism of thermal activation for both stages is the same or at least closely related. EBSD micrographs show that the occurrence of stage IV is related to a characteristic geometrically ordered cell-structure. At higher temperatures, steady state is reached due the balance between subgrain growth and deformation-induced formation of new subgrain boundaries. In combination with thermally activated yield stress prediction, this model, recently implemented in the MatCalc software package, offers a powerful tool for modeling and simulation of the stress-strain response of polycrystalline materials.

\section{References}

[1] G. Langford, M. Cohen, Strain hardening of Iron by severe plastic deformation, Trans. ASM 62 (1969) 623-638

[2] Ø. Ryen, H. I. Laukli, B. Holmedal, E. Nes, Large strain work hardening of aluminum alloys and the effect of mg in solid solution, Metall. Mater. Trans. A37 (2006) 2007-2013

[3] U. F. Kocks, Kinetics of solution hardening, Metall. Trans. A16 (1985) 2109-2129

[4] U. F. Kocks, Laws for Work-Hardening and Low-Temperature Creep, J. Eng. Mater. Technol. 89 (1976) 76-85

[5] E. I. Galindo-Nava, J. Sietsma, P. E. J. Rivera-Díaz-Del-Castillo, Dislocation annihilation in plastic deformation: II. Kocks-Mecking Analysis, Acta Mater. 60 (2012) 2615-2624

[6] S. Brinckmann, R. Sivanesapillai, A. Hartmaier, On the formation of vacancies by edge dislocation dipole annihilation in fatigued copper, Int. J. Fatigue 33 (2011) 1369-1375

[7] L. S. Tóth, A. Molinari, Y. Estrin, Strain Hardening at Large Strains as Predicted by Dislocation Based Polycrystal Plasticity Model, J. Eng. Mater. Technol. 124 (2002) 71-77

[8] E. Nes, Modelling of work hardening and stress saturation in FCC metals, Prog. Mater. Sci. 41 (1997) 129-193

[9] E. Nes, Recovery Revisited, Acta Metall. 43 (1994) 2189-2207 
[10]U. F. Kocks, H. Mecking, Physics and phenomenology of strain hardening: The FCC case, Prog. Mater. Sci. 48 (2003) 171-273

[11]D. Sparber, Dislocation substructure evolution of an Al-6016-T4 alloy during cold deformation, Diploma Thesis, TU Wien, 2016

[12]G. Schoek, The Activation Energy of Dislocation Movement, Phys. Status Solidi 8 (1965) 499-505

[13] N. Hansen, X. Huang, Microstructure and flow stress of polycrystals and single crystals, Acta Mater. 46 (1998) 1827-1836 